\title{
Hsp70 in mitochondrial biogenesis: From chaperoning nascent polypeptide chains to facilitation of protein degradation
}

\author{
R. A. Stuart, D. M. Cyr* and W. Neupert** \\ Institut für Physiologische Chemie der Universität München, Goethestrasse 33, D-80336 München (Germany)
}

\begin{abstract}
The family of hsp70 (70 kilodalton heat shock protein) molecular chaperones plays an essential and diverse role in cellular physiology. Hsp70 proteins appear to elicit their effects by interacting with polypeptides that present domains which exhibit non-native conformations at distinct stages during their life in the cell. In this paper we review work pertaining to the functions of hsp 70 proteins in chaperoning mitochondrial protein biogenesis. Hsp70 proteins function in protein synthesis, protein translocation across mitochondrial membranes, protein folding and finally the delivery of misfolded proteins to proteolytic enzymes in the mitochondrial matrix.

Key words. Mitochondrial biogenesis; nascent polypeptide chains; protein translocation; matrix-ATP; mitochondrial hsp 70; molecular chaperones.
\end{abstract}

\section{Introduction}

The life cycle of an eukaryotic protein includes its synthesis on ribosomes, sorting to a specific subcellular compartment, folding to an active conformation and finally its turnover. Different members of the hsp70 family of molecular chaperones have been observed to help facilitate each of these stages in protein metabolism ${ }^{19}$. The general mechanism for hsp70 protein function involves its ATP dependent binding to and release from polypeptides that assume non-native conformations ${ }^{23}$. Transient complex formation between hsp 70 and polypeptides appears to stabilize the conformation of substrate proteins and prevent their misfolding or aggregation. Upon release from hsp 70 different substrate proteins have different fates. For example, nascent polypeptide chains bound by hsp70 during protein synthesis often become substrates of other chaperones such as hsp60 and hsp10 proteins, which facilitates their folding to native conformations ${ }^{23}$. In other cases, nascent proteins which contain intracellular targeting signals are released from hsp70 and enter pathways for protein translocation into subcellular organelles ${ }^{44}$. In contrast, proteins damaged by physiological stress, and then bound by hsp 70 , are often delivered to the proteolytic machinery of the cell for degradation 9,16 .

Studies on the biogenesis of mitochondrial proteins have provided many of the seminal observations concerning the functions of hsp 70 proteins in these different aspects of protein metabolism. In this review we outline the basic steps of the mitochondrial protein import pathway and provide key examples where different hsp 70 proteins act to catalyze different reactions required for the biogenesis and turnover of mitochondrial enzymes. In this manner the reader is provided with a concise view of how interactions of hsp 70 with substrate proteins can facilitate a series of reactions that are fundamental to protein metabolism.

\section{The mitochondrial protein import pathway}

The majority of mitochondrial proteins are encoded by the nucleus and are synthesized on cytoplasmic ribosomes as precursor proteins. Studies with Saccharomyces cerevisiae indicate that assembly of nuclear encoded proteins into mitochondria requires the action of several different classes of cytosolic and mitochondrial molecular chaperones (table). Mitochondrial proteins are typically synthesized in a precursor form containing a transient amino-terminal presequence which serves as a targeting signal. Protein import into mitochondria normally occurs via a post-translational mechanism along a multistep pathway (fig. 1) which begins with the interaction of cytosolic hsp70 proteins (ct-hsp70) with nascent precursor proteins during their synthesis on polyribosomes $^{3,35}$. Interactions of hsp70 proteins with newly synthesized precursor proteins are assisted by the cytosolic DnaJ homolog Ydj1p ${ }^{6,13}$. By working together, hsp70 and DnaJ family members are thought to maintain precursor proteins in loosely folded conformations that are competent for passage across mitochondrial membranes ${ }^{6,15}$. Targeting of proteins to mitochondria involves the specific recognition of the presequence by receptor proteins located on the surface of the mitochondrial outer membrane ${ }^{29}$. This is followed by their translocation across the outer and inner mitochondrial membranes which is mediated by independent translocation machineries in the respective membranes ${ }^{29}$. Passage of the presequence into mitochondria is dependent on both a membrane potential, $\Delta \Psi$, across the inner membrane, and ATP 
Table. Hsp70 homologs and other molecular chaperones that assist in the biogenesis of mitochondrial proteins in $S$. cerevisiae. Ssa, Ssb and Ssc denote proteins from different hsp70 subfamilies. Ydj1p and Mdj1p are homologs of the E. coli protein DnaJ. Mgelp is a homolog of the E. coli protein GrpE. Hsp60 and hsp10 are heat shock proteins of 60 and $10 \mathrm{kd}$ in size, respectively.

\begin{tabular}{|c|c|c|c|}
\hline Chaperone & $\begin{array}{l}\text { Cellular } \\
\text { location }\end{array}$ & $\begin{array}{l}\text { Essential } \\
\text { for viability }\end{array}$ & Reference \\
\hline $\mathrm{Ssblp} / \mathrm{Ssb} 2 \mathrm{p}^{\mathrm{a}}$ & Cytosol & $\mathrm{No}^{\mathrm{b}}$ & Nelson et al. ${ }^{35}$ \\
\hline Ssa 1p/Ssa 2p & Cytosol & Yes & Deshaies et al. ${ }^{15}$ \\
\hline Ydjlp & Cytosol & $\mathrm{No}^{c}$ & $\begin{array}{l}\text { Caplan \& Douglas } \\
\text { Atencio \& Yaffe }\end{array}$ \\
\hline Ssclp (mt-hsp70) & $\begin{array}{l}\text { Mitochondrial } \\
\text { matrix }\end{array}$ & Yes & $\begin{array}{l}\text { Craig et al. }{ }^{10} \\
\text { Kang et al. }{ }^{28}\end{array}$ \\
\hline Mdjlp & $\begin{array}{l}\text { Mitochondrial } \\
\text { matrix }\end{array}$ & $\mathrm{No}^{\mathrm{d}}$ & Rowley et al. ${ }^{41}$ \\
\hline Mgelp & $\begin{array}{l}\text { Mitochondrial } \\
\text { matrix }\end{array}$ & Yes & Ikeda et al. ${ }^{27}$ \\
\hline Hsp60 & $\begin{array}{l}\text { Mitochondrial } \\
\text { matrix }\end{array}$ & Yes & Cheng et al. ${ }^{7}$ \\
\hline Hspl0 & $\begin{array}{l}\text { Mitochondrial } \\
\text { matrix }\end{array}$ & Yes & $\begin{array}{l}\text { Rospert et al. }{ }^{40} \\
\text { Höhfeld \& Hartl }\end{array}$ \\
\hline
\end{tabular}

${ }^{a} \mathrm{Ssb} 1 / \mathrm{Ssb} 2 \mathrm{p}$ are not specifically required for protein translocation into mitochondria, but are included here because they are proposed to interact with most nascent proteins during their synthesis on ribosomes.

${ }^{b}$ Deletion strains are cold-sensitive for growth.

${ }^{\circ}$ Deletion strains are viable at $23^{\circ} \mathrm{C}$, but not at $37^{\circ} \mathrm{C}$.

${ }^{d}$ Deletion strains are temperature-sensitive for growth on fermentable carbon sources and non-viable at all temperatures on nonfermentable carbon sources.

hydrolysis in the matrix. Upon entry into the matrix, the presequence is cleaved from the precursor protein by the matrix processing peptidase (MPP). Complete translocation of precursors into the matrix is dependent on ATP and the mitochondrial hsp70 cognate, Ssclp (mt-hsp70: see ref. 18, 43). Upon completion of translocation into the matrix, mt-hsp70 and the mitochondrial DnaJ homolog Mdjlp act on the precursor protein to facilitate its folding to an active conformation $^{41}$. These proteins may act alone or in combination with hsp60 and hsp10 to facilitate this reaction (fig. 1). Finally, after the productive life of a mitochondrial protein is over, $\mathrm{mt}-\mathrm{hsp} 70$ and Mdjlp also facilitate the delivery of misfolded or

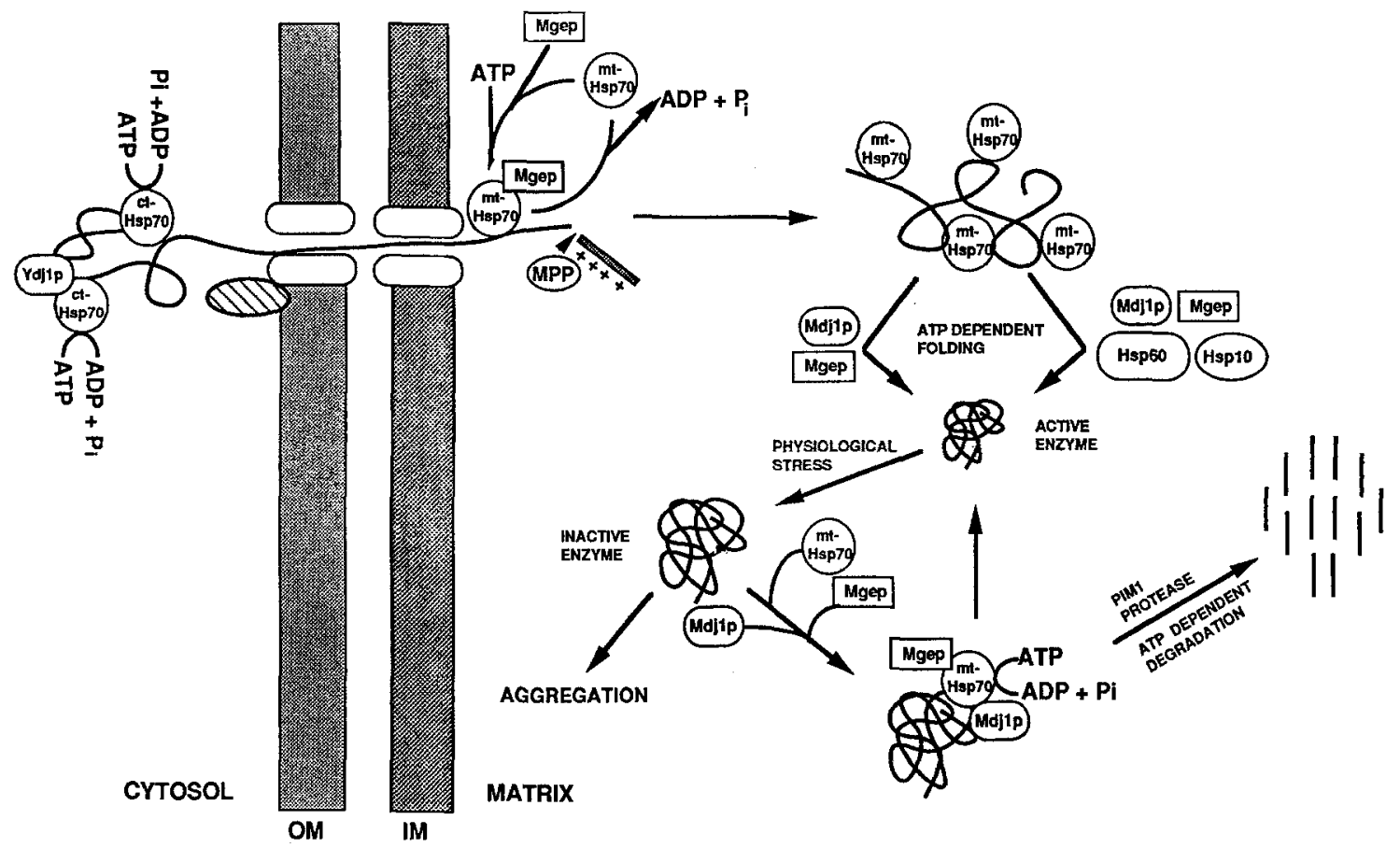

Figure 1. Hypothetical model for the functions of hsp70 homologs in the biogenesis of mitochondrial proteins. Ct-hsp 70 represents the Ssa1 and Ssa2 proteins. MPP denotes the matrix processing protease responsible for clevage of the mitochondrial presequence. The presequence is represented by the shaded and positively charged region of the precursor protein. PIM1 is an ATP dependent protease found in the matrix of mitochondria. See table for the definitions to the other terms used in this figure. 
denatured proteins to the protein degradation machinery of mitochondria ${ }^{53}$. Thus, hsp 70 proteins located in the cytosol and mitochondrial matrix chaperone many different stages in the life cycle of a mitochondrial protein. Details concerning the functions of different hsp70 family members, in particular mitochondrial hsp70 (mt-hsp70), in these different reactions will be provided below.

\section{Interactions of cytosolic hsp70 family members with nascent precursor proteins}

The cytosol of $S$. cerevisiae contains two different subfamilies of hsp70 proteins, the Ssa and Ssb proteins (table). These different subfamilies of hsp70 proteins exhibit about $60 \%$ identity to each other, but play different roles in protein metabolism ${ }^{9}$. The Ssb hsp70 proteins interact with nascent, presumably unfolded, polypeptides early in their synthesis, possibly as they emerge from the large subunit of the ribosome, and help facilitate the elongation of polypeptide chains ${ }^{35}$. The Ssa hsp70 proteins also interact with nascent polypeptides, but apparently at a later stage in protein synthesis, after they have assumed some aspect of secondary structure ${ }^{3,8}$. Interaction of the Ssa hsp70 proteins with mitochondrial precursor proteins has been shown to help facilitate their translocation into mitochondria ${ }^{15}$. The Ssb hsp70 proteins on the other hand, do not appear to be required for protein translocation into mito-

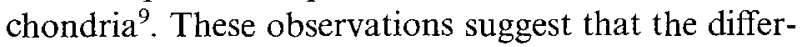
ent subfamilies of cytosolic hsp 70 proteins interact with the same substrate proteins in sequential order with the Ssb proteins binding first, followed by the Ssa proteins. Interaction with the Ssb proteins would be important for the rapid synthesis of mitochondrial precursors or other proteins, while interaction with the Ssa proteins would assure that proteins are delivered to mitochondria in a transport competent conformation.

What mechanism determines the order by which these different cytosolic hsp70 proteins act? One possibility is that the Ssa and Ssb hsp70 proteins have different substrate specificities ${ }^{9}$. The Ssb proteins might preferentially bind extended polypeptides as they emerge for ribosomes, whereas the Ssa hsp70 proteins could prefer partially folded polypeptides formed later during protein synthesis. The binding and release of some partially folded substrates to hsp 70 proteins is assisted by the action of the $E$. coli DnaJ protein and its homologs ${ }^{13}$. The cytosol of $S$. cerevisiae contains at least two different DnaJ homologs, one of which is Ydj1 ${ }^{5}$. Ydj1p has been shown to specifically interact with Ssa1p and Ssa2p, but not the Ssb proteins, to regulate their chaperone function ${ }^{11,12}$. Ydj1p appears to function with the Ssa hsp 70 proteins in protein translocation events. Mutations in Ydjlp cause defects in mitochondrial protein import ${ }^{2,6}$. Ydj1p may serve to facilitate the binding of partially folded precursor proteins to Ssa hsp70 proteins and thereby improve their ability to be translocated across membranes. In addition, the specificity observed for the interactions between Ydjlp and the Ssa and Ssb hsp70 proteins may assist in determining the order in which these proteins interact with nascent precursor proteins.

In spite of the above discussion, it should be noted that the requirement for cytosolic hsp 70 proteins in protein translocation across mitochondrial membranes is not universal. A recent study from the Schatz laboratory has compared the cytosolic ATP/ hsp70 requirements for protein translocation into mitochondria ${ }^{52}$. They observe that only a subset of mitochondrial precursor proteins require cytosolic ATP to maintain their translocation competence. Proteins that require cytosolic ATP for import appear to be prone to aggregation or misfolding and require cytosolic hsp70 proteins to prevent this from occurring prior to the import event. However, precursor proteins not prone to nonspecific reactions are imported efficiently in the absence of ATP dependent chaperone function in the cytosol ${ }^{52}$. In contrast, the import of all matrix proteins requires the action of mt-hsp $70^{43,52}$. The details of mt-hsp70 function in protein translocation across the membranes of mitochondria will be discussed in the sections below.

\section{Mt-hsp70 facilitates several aspects of protein translocation across the inner membrane of mitochondria}

Recent results from two independent lines of experiments have shed light on the importance of mt-hsp 70 for protein import into mitochondria, as it appears to perform essential functions at several distinct steps of the import pathway ${ }^{14,18,20,28,43,50}$. These experiments were specifically designed to modulate the activity of $\mathrm{mt}$-hsp70 and using a powerful in vitro import system, to directly address the consequences of such manipulations on protein import. Two experimental approaches were used, namely, 1) modulation of matrix ATP concentrations to levels that adversely affect the ATP-dependent action of mthsp70 and 2) mutations in the SSC1 gene, encoding $\mathrm{mt}-\mathrm{hsp} 70$ in $S$. cerevisiae, resulting in mutated mthsp70 proteins which are temperature sensitive (ts) for function.

In isolated mitochondria matrix ATP levels can be easily manipulated using a combination of oligomycin, a specific inhibitor of the ATP synthase, to prevent new ATP synthesis and carboxyatracty- 
loside, which blocks the transport of exogenously added ATP into the mitochondrial matrix at the level of the ADP/ATP carrier. By doing so the levels of matrix ATP can be stringently reduced to experimentally definable levels, independent of affecting the $\Delta \Psi$ (membrane potential). In vitro protein import studies using such 'ATP-depleted mitochondria' make it possible to find out which specific stages of the import process are blocked when matrix ATP concentrations become limiting for mt-hsp70 action $^{14,20,43}$

Genetic manipulation of mt-hsp70 action was also achieved following the production of ts yeast mutants carrying mutations in the SSC1 gene $\mathrm{g}^{18,28,50}$. Two such mutants have been characterized, ssc $1-2$ and $\operatorname{ssc} 1-3$, both of which displayed an inhibition of protein import into mitochondria in vivo when the cells were shifted to the non-permissive temperature. Such mutants have proved invaluable for the study of mt-hsp70 action as their import ts phenotype can be induced in vitro, when isolated mitochondria from the mutant strains are exposed to non-permissive temperature prior to import studies. The ssc1-2 mutation mapped to the putative peptide binding domain at the carboxy-terminus of mt-hsp70. Mthsp70 from the ssc1-2 mutant displayed a very strong binding activity to substrates, however, it was proposed that the subsequent release from substrate was somehow affected. The ssc1-3 mutation, on the other hand, mapped to the amino-terminal ATPase domain of $\mathrm{mt}$-hsp 70 and resulted in a stronger defect in protein import than observed for the ssc $1-2 \mathrm{mu}-$ tant (see below). The observed in vitro import phenotype with the $s s c 1-3$ mitochondria was consistent with the suggestion that mt-hsp 70 activity was compromised at the early level of initial binding to incoming precursor proteins.

The results stemming from both, the study of the energetics of protein import and the in vitro analysis of the ts $\operatorname{ssc} 1$ mutants, have indicated an involvement of mt-hsp70 at at least three distinct stages of mitochondrial import ${ }^{36,44}$ : 1) Mt-hsp70 interacts with mitochondrial targeting sequences upon their spontaneous exposure to the matrix and thereby stabilizes them on the trans side of the inner membrane. This important mt-hsp70 action serves to make the initial import step irreversible and represents the first step of commitment for the precursor in the import process. 2) Mt-hsp70, by binding to matrix exposed parts of preproteins, serves to secure the unfolding of tightly-folded segments of preproteins on the cis side of the outer membrane. 3) Through a series of binding and release cycles, involving additional domains of the preprotein, mt-hsp70 action is required for completion of translocation across the inner membrane.
In the following section we wish to summarize the experimental evidence which has given rise to these conclusions for the action of $\mathrm{mt}-\mathrm{hsp} 70$.

\section{Stable translocation of mitochondrial targeting presequence requires mt-hsp70 binding}

The majority of mitochondrial precursor proteins carry amino-terminal cleavable presequences. Such presequences perform multiple important roles during import. They are initially responsible for targeting the precursor to the mitochondria where it interacts with the mitochondrial receptor import complex of the outer membrane. Upon insertion across the outer membrane, the presequence targets the precursor to an independent inner membrane translocation apparatus. Finally, the spontaneous passage of the presequence across the inner membrane, in response to $\Delta \Psi$, initiates the translocation of the precursor across the inner membrane. It appears now that this initial passage of the presequence across the inner membrane is a reversible event and is rendered permanent only when its presence is stabilized in the matrix by an ATP-dependent reaction, possibly by binding to $\mathrm{mt}-\mathrm{hsp} 70^{14}$.

When mitochondrial matrix ATP was experimentally depleted to extremely low levels, a complete inhibition of precursor import was observed. More interestingly, the precursor proteins accumulated as unprocessed species on the outer surface of the mitochondria, accessible to exogenously added protease ${ }^{14}$. These unprocessed precursors, which accumulated on the mitochondrial surface, represented true translocation intermediates, as they could be further chased into the mitochondria in a second independent reaction. This chase reaction required not only the restoration of high levels of ATP in the matrix but also required the presence of $\Delta \Psi$ across the inner membrane. The identification of such an intermediate implicated a role for mt-hsp70 at this early stage of import. Thus it was proposed that stable presequence translocation across the inner membrane requires the concerted action of both the $\Delta \Psi$ and the function of mt-hsp70. In the absence of mt-hsp70 action, but in the presence of $\Delta \Psi$, the presequence becomes translocated across the inner membrane, where once exposed to the matrix it may undergo processing by MPP. Under these circumstances, however, neither the processed nor the unprocessed precursor is stably exposed to the matrix, unless it is anchored there through the direct binding to mt-hsp70. In the absence of this occurring, the initial translocation step is reversible and the polypeptide can slip back to the cis side of the inner membrane. Upon doing so, presumably, the unprocessed species accumulates at the level of the outer membrane translocation machinery ${ }^{14}$. 
Thus, mt-hsp 70, by binding to the incoming polypeptide upon exposure to the matrix, makes this initial import step unidirectional by preventing the polypeptide from slipping back across the inner membrane and even out of the mitochondria. It is not yet clear whether the binding of mt-hsp 70 occurs through direct interaction with the presequence or with amino-terminal mature regions of the precursor. Furthermore, the extent to which a precursor can be cleaved in the matrix in the apparent absence of mt-hsp70 activity was observed to vary from precursor to precursor and most probably reflects the stability and depth to which the precursor can initially insert across the inner membrane.

\section{Mt-hsp70 activity is required for unfolding of precursors outside the mitochondria}

On the basis of a number of observations it is generally agreed that precursor proteins cannot cross membranes in a folded conformation. These findings include 1) when precursors were bound to antibodies, import was completely abolished ${ }^{42}$;2) fusion proteins consisting of mitochondrial presequences fused to mouse dihydrofolate reductase (DHFR) were not imported into mitochondria when the DHFR moiety was stabilized through the binding of a ligand, methotrexate $^{17}$; and 3) specially designed fusion proteins were used to map the minimum polypeptide length required to concomitantly span both the outer and inner membrane. Approximately 50 amino acid residues were found to be required to span such a distance $^{39}$. Considering all of these observations, the data suggest that the incoming precursor spans the import channels as an extended polypeptide chain.

In the cytoplasm, precursor proteins destined for import do not generally adopt their final functional conformation. This folding is thought to be prevented by a number of factors; for example, the potential hinderance of complete folding by the targeting signal or presequence, the lack of bound prosthetic groups which sometimes form an integral part of the mature functional enzyme and of course, as discussed previously, the presence of cytosolic chaperones which interact with precursors and by doing so are thought to prevent unfavorable folding reactions. On the other hand, it is clear that at least domains of some preproteins do display the tendency to tightly fold whilst in the cytoplasm and yet these preproteins are imported efficiently into mitochondria ${ }^{20}$. How does the import process cope with such folded domains? Does a mechanism exist to 'unfold' such domains? Recently it has become clear that mt-hsp70 plays an important role in this process of preprotein unfolding.
During the course of studying the energy requirements of the import of the precursor of cytochrome $b_{2}\left(\mathrm{p} b_{2}\right)$, it was demonstrated that mt-hsp 70 activity is required for the unfolding of tightly-folded segments in the mature part of the precursor outside the mitochondria. Cytochrome $b_{2}$ (L-lactate dehydrogenase) is located in the intermembrane space and contains both heme and flavin as prosthetic groups. The initial 100 amino acid residues of the mature cytochrome $b_{2}$ polypeptide chain (corresponding to amino acid 81-181 of the precursor) constitute a tightly-folded structure, termed the cytochrome $b_{5}$ or heme binding domain ${ }^{54}$. This folded domain also has been shown to occur in the precursor protein prior to import ${ }^{20}$.

The import of cytochrome $b_{2}$ displayed a very strong requirement for matrix $\mathrm{ATP}^{20,26,43}$. In the absence of matrix ATP, the precursor accumulated as an unprocessed species on the outer surface of mitochondria. Very similar results were obtained if the import was performed using mitochondria prepared from the $s s c 1-3$ mutant $t^{50}$. Thus the necessity for matrix ATP for cytochrome $b_{2}$ import reflected a dependence on mt-hsp70. Cytochrome $b_{2}$-derived preproteins, consisting of 185 or more residues of $\mathrm{p} b_{2}$ fused to DHFR, displayed the same dependence on matrix ATP and mt-hsp70 function. In contrast, the import of shorter fusion proteins (up to 167 residues of $\mathrm{p} b_{2}$ ) was largely independent of matrix $\mathrm{ATP}^{20}$. Furthermore, if the precursors of cytochrome $b_{2}$ and those $\mathrm{p} b_{2}$-derived fusion proteins, which displayed strong matrix ATP/mt-hsp70 requirements, were denatured in $8 \mathrm{M}$ urea before import, they could be imported very efficiently into both ATP-depleted mitochondria and into sscl-3 mutant mitochondria $^{20,43,50}$

These findings are consistent with the suggestion that if the cytochrome $b_{5}$ domain is intact in a $\mathrm{p} b_{2}$ derived precursor protein and folds into a stable structure, it can prevent import of the precursor unless it becomes unfolded, a process requiring mthsp70 activity. The presence of this folded domain so close to the amino terminus most probably prevents the stable insertion of the presequence into the matrix, unless it becomes unfolded through the action of mt-hsp70. Hence all precursors with this domain require ATP-dependent binding of mt-hsp70 to matrix exposed parts of the protein in order to mediate its unfolding outside the mitochondria. By contrast, cytochrome $b_{2}$ constructs in which this domain was truncated ( $\mathrm{p} b_{2}$ 167-DHFR and smaller) or disrupted ( $\left.\mathrm{p} b_{2} \Delta 135-226\right)$ can be processed efficiently and imported in the apparent absence of mt-hsp70 activity. In summary, these data suggest a function of $\mathrm{mt}-$ hsp70 in supporting the unfolding of precursor on the mitochondrial surface. A possible mechanism is 
that, by binding to segments of precursor on the matrix side, stabilization of an unfolded conformation on the mitochondrial surface is favored.

\section{Mt-hsp70 activity is required for the completion of preprotein translocation into mitochondria}

In addition to stabilizing the presequence of precursors in the mitochondrial matrix, the binding of mt-hsp 70 to incoming polypeptide chains is essential for both precursor unfolding and completion of translocation across the membrane system ${ }^{36,44}$. Completion of import in contrast to translocation of the presequence does not require $\Delta \Psi$, but only requires ATP hydrolysis. That this ATP hydrolysis reflects a requirement for $\mathrm{mt}-\mathrm{hsp} 70$ activity is supported by findings with the ssc 1-2 mutant ${ }^{28}$. When mitochondria from this mutant were incubated at the non-permissive temperature, MPP processing of preproteins could be observed. However, these precursors failed to become completely imported as they remained accessible to exogenously added proteases and accumulated as translocation intermediates spanning both mitochondrial membranes. Such intermediates existed in a complex with mt-hsp70 as they could be co-immunoprecipitated with $\mathrm{Ssclp}$ specific antibodies ${ }^{28}$.

Completion of translocation thus requires mt-hsp70 activity. Upon exposure to the matrix, mt-hsp70 initially binds to amino-terminal parts of the protein to secure the preprotein and thereby render this initial step of import unidirectional. Spontaneous relaxing or breathing (partial unfolding) of the remaining parts of the preprotein outside the mitochondria renders further segments free to pass across the translocation channels. Upon their exposure to the matrix, mt-hsp70 binds to them, preventing their ability to slip back and thus committing them irreversibly to the matrix. Hence the preprotein is translocated in a step-wise manner where further binding of $\mathrm{mt}$-hsp 70 commits increasing amounts of the preprotein to the matrix. By doing so, the equilibrium of the preprotein structure outside the mitochondria is shifted towards the unfolded state which in turn enables it to be drawn across the membranes, ultimately resulting in its accumulation within the matrix.

\section{Folding of newly imported proteins is mediated by chaperones in the matrix}

Preproteins are translocated across the mitochondrial membrane system as extended polypeptide chains and once in the matrix must fold correctly to attain their native functional conformation. In many cases this folding process is an assisted one, involving the cooperation of mt-hsp 70 and sometimes transfer to another matrix-localized chaperone, hsp $60^{7,28,34,37}$.
Evidence for the involvement of mt-hsp70 in protein folding came initially from in vitro studies with the ssc 1-2 mutant mitochondria. The import defect observed in these mitochondria could be overcome if the precursors were denatured in $8 \mathrm{M}$ urea prior to import. Although the precursors became imported to a protease resistant location, they were found unfolded in the matrix and remained bound to $\mathrm{mt}-\mathrm{hsp} 70^{28}$.

In bacterial systems the function of DnaK, the homologue of mt-hsp70, is regulated by two other proteins, DnaJ and $\mathrm{GrpE}^{31,32}$. Recently mitochondrial homologues of these two proteins have been found in $S$. cerevisiae. The mitochondrial DnaJ homologue, termed Mdj1p, is localized on the matrix-side of the inner membrane ${ }^{41}$. Mdj1p is not an essential protein, but deletion of the MDJl gene ( $\Delta$ mdj1 strain) results in a ts growth phenotype and respiratory-defective mitochondria deficient in mitochondrial DNA. Precursor proteins were efficiently imported into mitochondria prepared from the $\Delta$ mdj1 strain, however, folding of newly imported proteins and their stability against heat denaturation were reduced ${ }^{41}$. Though not essential for folding and refolding, the presence of Mdj1p clearly makes these processes more efficient, especially at elevated temperatures.

A mitochondrial GrpE homologue, Mgelp, has also been reported recently ${ }^{27}$. Like Mdjlp, Mgelp is a matrix-localized protein; however, in contrast it is an essential protein, since deletion of the MGE1 gene was lethal to the cell. The function of Mgel $p$ in both import and folding processes in mitochondrial assembly is presently unknown and awaits further characterization of this protein.

Folding of proteins mediated by hsp60 in the matrix is an ATP-dependent process ${ }^{7,37}$. In order to become folded by this chaperone, proteins following import must be transferred from mt-hsp 70 to hsp60, a process inhibited in both the ssc1-2 mutant and apparently in the $\Delta$ mdj 1 mutant (ref. 28; Stuart, T. Langer \& Neupert, unpubl. observ.). Hsp60 belongs to a class of highly conserved proteins, termed chaperonins which are found wide-spread in both prokaryotes (GroEL in E. coli) and eukaryotes. Hsp60 is a homo-oligomeric protein composed of 14 subunits of molecular weight of $60 \mathrm{kDa}$. The activity of hsp60 is modulated by a co-chaperonin, hsp 10, homologous to GroES in E. coli. A mitochondrial homologue of GroES has been identified recently in yeast mitochondria ${ }^{40}$.

\section{Mt-hsp70, a molecular chaperone for proteins encoded by mitochondrial DNA}

In the yeast $S$. cerevisiae, the vast majority of mitochondrial proteins are nuclear encoded, whilst only eight proteins are encoded by the mitochondrial 


\section{Aggregation}

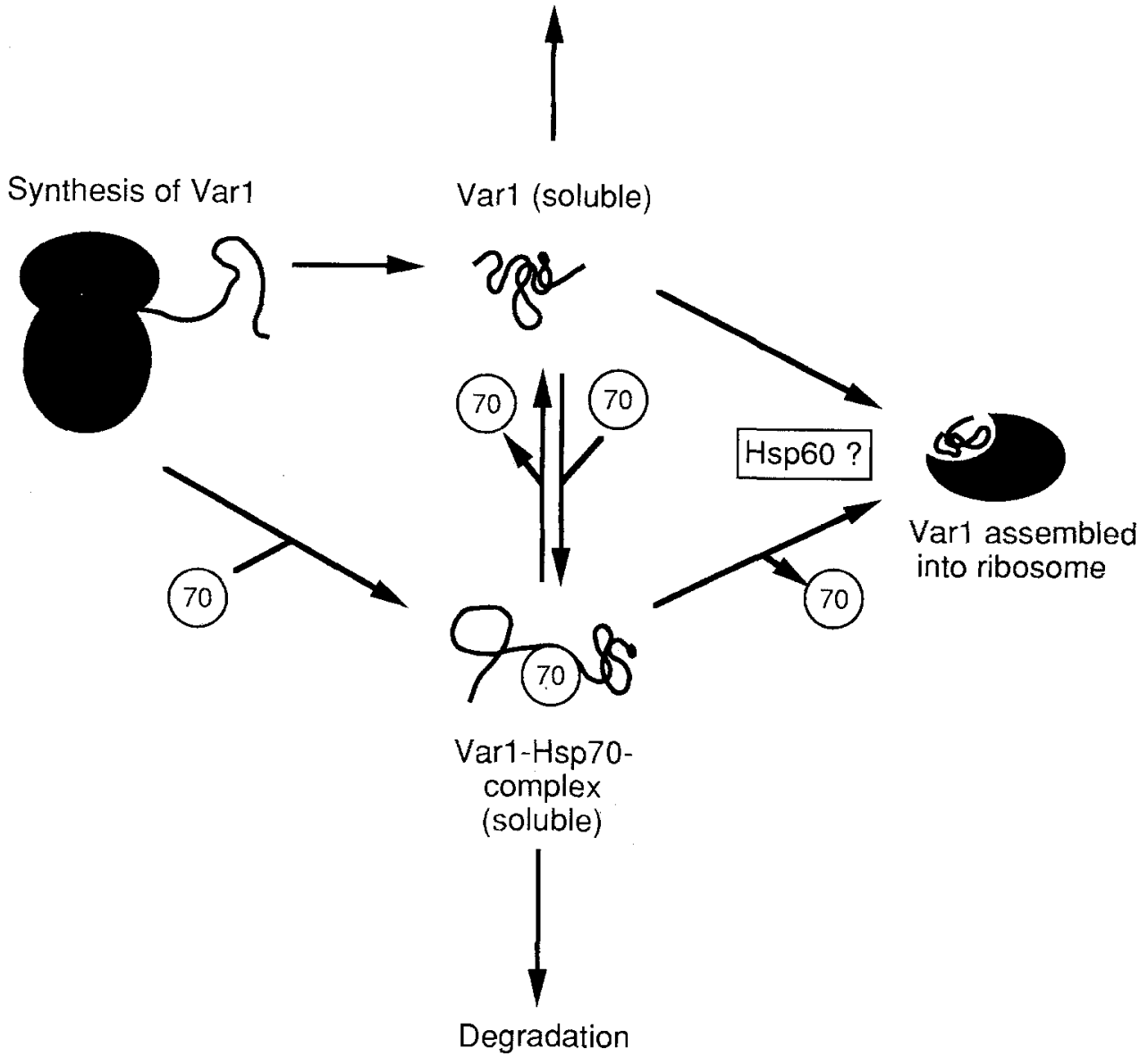

Figure 2. Current model for the role of mt-hsp 70 in the biogenesis of proteins encoded by mitochondrial DNA. This model represents reactions that take place in the mitochondrial matrix. Varl is a mitochondrially encoded protein that is assembled into the small subunit of mitochondrial ribosomes. 70 denotes mt-hsp70. The reactions involving hsp 70 in the matrix are thought to all require the hydrolysis of ATP, which is not shown here to simplify the model.

genome $\mathrm{e}^{4,47}$. These proteins are cytochrome $b$ of the cytochrome $b c_{1}$ complex, subunits 6,8 and 9 of the $F_{0}$ part of the $F_{1} F_{0}$-ATP synthase (ATPase6, ATPase8 and ATPase9, respectively), cytochrome oxidase subunits I, II and III (COXI, COXII and COXIII, respectively) and finally the var1 protein, a component of the ribosomal small subunit ${ }^{21,22,46}$. Hence all of these proteins are subunits of larger oligomers and their assembly into functional complexes requires the coming together of cytosolically synthesized subunits which have been imported into the mitochondria with these proteins which have been synthesized in the matrix. In a recent study addressing the assembly of these mitochondrially encoded subunits, we presented evidence for a new role of mt-hsp70, namely, as a chaperone for newly synthesized proteins encoded by the mitochondrial genome ${ }^{23 a}$. In particular, it was proposed that mt-hsp70 functions to prevent misfolding and hence aggregation of at least some of the newly synthesized mitochondrially encoded proteins (fig. 2). This mech- anism would ensure the efficient folding and assembly of mitochondrial proteins.

By studying mitochondrial protein translation in the ssc $1-2$ and $s s c 1-3$ ts mutants, it could be concluded that the availability of $\mathrm{mt}-\mathrm{hsp} 70$ function influences the pattern of proteins synthesized both in vivo and in vitro. In addition $\mathrm{mt}-\mathrm{hsp} 70$ interacted directly with some of the mitochondrial translation products and thereby supported their subsequent assembly, in particular under thermal stress conditions. By interacting with these newly synthesized proteins, mthsp70 could prolong the life-time of the assembly competent state of the protein. In this study, the role of $\mathrm{mt}$-hsp70 in the assembly process of varl and of two subunits of the $F_{1} F_{0}$-ATP synthase, ATPase6 and ATPase9, was focused upon ${ }^{23 a}$

The var1 protein is the only hydrophilic non-membrane protein encoded by the mitochondrial genome in $S$. cerevisiae. It is thought to be synthesized on soluble mitochondrial ribosomes and assembled into pre-ribosomal complexes whose protein content is 
entirely nuclear encoded. Newly synthesized varl in vitro existed initially as a soluble protein in the matrix, from there its destiny was determined by a number of factors, namely the availability of pre-ribosomal complexes, presence of functional mt-hsp 70 and the activity of the mitochondrial proteolytic degradation system ${ }^{23 a}$. If the assembly process of varl into the pre-ribosomal complexes was limiting, i.e. due to insufficient numbers of complexes, the remaining unassembled varl protein existed as a soluble species in the matrix, and could be shown to be stabilized through a direct interaction with mt-hsp70. When mt-hsp70 activity was limiting however, for example, in the ssc 1 ts mutants, the unassembled var 1 displayed a tendency to aggregate. This aggregation was particularly pronounced at elevated temperatures, thus indicating that a physical interaction with mt-hsp70 was particularly necessary under these stress conditions. The formation of such aggregates had a significant effect on the solubility and assembly competence of the varl protein.

Furthermore, in wild-type mitochondria, the unassembled var1 appeared to be susceptible to proteolytic degradation. This degradation process, however was not observed in the ssc 1 mutants, suggesting that it requires a functional mt-hsp 70 to facilitate it. It is not yet clear which protease actually catalyses this degradation process, although the recently identified PIM1 protease may be involved ${ }^{30,49}$. Proteolytic degradation by this protease was recently found to be closely coupled to mt-hsp70 activity (ref 53; see below).

In summary it appears that mt-hsp70 functions as a molecular chaperone to prevent misfolding of the unassembled var1 and thereby ensures efficient ribosomal assembly, particularly under stress conditions. In addition to newly synthesized var1, both monomers and oligomers of ATPase9 were found to physically interact with $\mathrm{mt}$-hsp70. Further investigation into the assembly of the ATP synthase in the ssc1 mutants suggested that, like the assembly of the var1, it too was supported by mt-hsp 70 activity. Following mitochondrial protein synthesis, the formation of a number of high molecular weight complexes could be observed. Two of the major complexes, a $48 \mathrm{kDa}$ and a $54 \mathrm{kDa}$ complex could be shown to be composed of subunits of the Fo part of the ATP synthase. The 48 kDa complex represents an ATPase 9 oligomer, whilst the $54 \mathrm{kDa}$ complex contains, in addition to subunits of ATPase9, the ATPase6 subunit. The $48 \mathrm{kDa}$ oligomer, in contrast to the $54 \mathrm{kDa}$ oligomer, could be found complexed to mt-hsp70. Further investigation revealed that the $48 \mathrm{kDa}$ oligomer represented an assembly intermediate of the ATP synthase whose interaction with $\mathrm{mt}-\mathrm{hsp} 70$ was necessary for the formation of a later assembly intermediate; i.e. the 54
$\mathrm{kDa}$ complex. Analysis of the formation of these two complexes in ssc1 mutant mitochondria demonstrated that the assembly of ATPase 6 into the $48 \mathrm{kDa}$ complex, resulting in the formation of the $54 \mathrm{kDa}$ complex was influenced adversely by the apparent absence of mt-hsp 70 action. As a consequence of the inhibition of ATPase6 assembly in the ssc 1 mutant mitochondria, this subunit was rapidly degraded, in contrast to the wild-type situation, where it becomes assembled and is thereby stable against degradation $^{23 a}$.

In summary, we have described a novel role for mt-hsp70, namely as a chaperone for at least three mitochondrial gene products. This role comprises 1) maintenance of a soluble state of unassembled proteins; 2) prevention of aggregation and 3) facilitation of assembly of newly synthesized proteins into larger complexes. Other possible functions of $\mathrm{mt}-$ hsp70 include mediating turnover of unassembled proteins by proteolytic degradation.

\section{Mt-hsp70 and the mitochondrial proteolytic degradation system}

Protecting cells against the damaging effects of stress conditions is another function of the hsp70 family $^{1,33}$. Physiological stress conditions, such as elevated temperatures, often compromise the structural integrity of proteins, resulting in their denaturation in the cell. Hsp70 proteins, whose expression is induced under stress conditions, bind to such damaged proteins thus preventing their aggregation, and support their efficient refolding to their native structure when conditions are more favorable ${ }^{19,25,38}$. Stabilization of such partially folded proteins by hsp70 also could facilitate their proteolytic degradation when their renaturation cannot be achieved. Recent evidence summarized below has demonstrated that the activity of the mitochondrial degradation system is intimately coupled with that of mt-hsp $70^{53}$. More specifically, it appears that a protein destined to be degraded must be kept in a soluble state in order to be recognized as a substrate for proteolysis. Maintenance of such a state, in turn, requires mt-hsp70 activity. Thus, molecular chaperones are postulated to act in a coordinated fashion with some proteases to rid the cell of proteins that are irreversibly damaged.

The proteolytic system of mitochondria, in contrast to the various functions of $\mathrm{mt}-\mathrm{hsp} 70$, is poorly characterized and understood. Recently, an ATP-dependent protease located in the mitochondrial matrix was described in both mammalian and yeast cells $\mathrm{s}^{30,45,49,51}$. Cloning of the respective gene revealed that the proteases were highly homologous to the bacterial La protease. In yeast, this ATP-dependent protease is 
encoded for by the PIM1 gene and deletion of this gene results in respiratory deficient mitochondria ${ }^{45,49}$. Furthermore, expression of this gene, similar to that of its prokaryotic homologue, is induced upon heat shock, suggesting that this protease may be responsible for the degradation of misfolded proteins formed under stress conditions. As discussed previously, following its synthesis in mitochondria, the unassembled varl protein was observed to be susceptible to proteolytic degradation. This proteolysis event was not observed in the ssc 1 mutant mitochondria, in contrast to wild-type, thus suggesting a functional link between mt-hsp 70 activity and the mitochondrial proteolytic system responsible for the degradation of this protein.

A study was undertaken recently to directly test the possible link between mt-hsp70 activity and proteolytic degradation of misfolded proteins. The role of molecular chaperones in facilitating the degradation of newly imported misfolded proteins, together with the PIM1 protease, was studied ${ }^{53}$. Proteolysis of these proteins, which was ATP-dependent and was mediated by the PIMl protease, was observed. Both mt-hsp70 and Mdjlp were required for the degradation. These molecular chaperones were found to be necessary to prevent the aggregation of the misfolded proteins and thus maintain them as soluble substrates, enabling their proteolysis. Mdj1 $\mathrm{p}$ was postulated to function by ensuring efficient binding of the polypeptide chains to $\mathrm{mt}-\mathrm{hsp} 70$. Release from $\mathrm{mt}-$ hsp70 was demonstrated to be a prerequisite for degradation by the PIM1 protease. This release step is promoted by Mdjlp.

In summary, these two molecular chaperones, together with PIM1, act in a concerted manner to ensure removal of misfolded proteins from the matrix. Thus, mt-hsp70 and Mdjlp were identified as essential components of the mitochondrial proteolytic degradation machinery.

Acknowledgments. Work described here from the laboratory of the authors was supported by the 'Deutsche Forschungsgemeinschaft' through SFB 184, the 'Fonds der Chemischen Industrie', the 'Münchner Medizinischen Wochenschrift' to R.A. Stuart and by a fellowship from the Human Frontier and Science Program to D. M. Cyr.

* Supported by a long-term fellowship from the Human Frontier Science Program Organization.

* To whom correspondence should be addressed.

1 Ang, D., Liberek, K., Skowyra, D., Zylicz, M., and Georgopoulos, C., Biological role and regulation of the universally conserved heat shock proteins. J. biol. Chem. 266 (1991) $24233-24236$

2 Atencio, D.P., and Yaffe, M.P., Mas5, a yeast homolog of DnaJ involved in mitochondrial protein import. Molec. cell. Biol. 12 (1992) 283-291.
3 Beckman, R.P., Mizzen, L., and Welch, W., Interaction of hsp70 with newly synthesized proteins: Implications for protein folding and assembly. Science 248 (1990) 850-856.

4 Borst, P., and Grivell, L.A., The mitochondrial genome of yeast. Cell 15 (1978) 705-723.

5 Caplan, A.J., and Douglas, M.G., Characterization of YDJ1; A yeast homolog of the E. coli dnaJ gene. J. Cell Biol, 114 (1991) 609-622.

6 Caplan, A.J., Cyr, D.M., and Douglas, M.G., YDJ1 facilitates polypeptide translocation across different intercellular membranes by a conserved mechanism. Cell 71 (1992) $1143-$ 1155.

7 Cheng, M.Y., Hartl, F.U., Martin, J., Pollock, R.A., Kalusek, F., Neupert, W., Hallberg, E.M., Hallberg, R.L., and Horwich, A. L., Mitochondrial heat-shock protein hsp60 is essential for assembly of proteins imported into yeast mitochondria. Nature 337 (1989) 620-625

8 Chirico, W.J., Dissociation of complexes between $70 \mathrm{Kda}$ stress proteins and presecretory proteins is facilitated by a cytosolic factor. Biochem. biophys. Res. Commun. 189 (1992) $1150-1156$

9 Craig, E.A., Baxter, B.K., Becker, J., Halladay, J., and Zeigelhoffer, T., Cytosolic hsp70s of Saccharomyces cerevisiae: Roles in protein synthesis, protein translocation, proteolysis and regulation, in: The Biology of Heat Shock Proteins and Molecular Chaperones, pp. 31-52. Eds R.I. Morimoto, A. Tissieres and C. Georgopoulos. Cold Spring Harbor Laboratory Press, Cold Spring Harbor, 1994

10 Craig, E.A., Kramer, S., Shilling, J., Werner-Washburne, M., Holmes. S., Kosic-Smither, J., and Nicolet., C.M., SSCl, an essential member of the $S$. cerevisiae HSP70 multigene family, encodes a mitochondrial protein. Molec. cell. Biol. 9 (1989) $3000-3008$

11 Cyr, D.M., and Douglas, M.G., Differential regulation of hsp70 subfamilies by the eukaryotic DnaJ homolog YDJ1. J. biol. Chem. 269 (1994) 9798-9804.

12 Cyr, D.M., Lu, X., and Douglas, M.G., Regulation of eukaryotic hsp70 function by a DnaJ homolog. J. biol. Chem. 267 (1992) 20927-20931.

13 Cyr, D.M., Langer, T., and Douglas, D.M., DnaJ-like proteins: molecular chaperones and specific regulators of hsp70. Trends biochem. Sci. 19 (1994) 176-181.

14 Cyr, D.M., Stuart, R.A., and Neupert, W., A matrix ATP requirement for presequence translocation across the inner membrane of mitochondria. J. biol. Chem. 268 (1993) 23751 23754.

15 Deshaies, R.B., Koch, B., Werner-Washburne, M., Craig, E.A., and Schekman, R., A subfamily of stress proteins facilitates translocation of secretory and mitochondrial precursor polypeptides. Nature 332 (1988) 800-805.

16 Dice, J.F., Agarraberes, F., Kirven-Brooks, M., Terlecky, L.J., and Terlecky, S.R., Heat shock 70-Kd proteins and lysosomal proteolysis, in: The Biology of Heat Shock Proteins and Molecular Chaperones, pp. 137-152. Eds R.I. Morimoto, A. Tissieres and C. Georgopoulos. Cold Spring Harbor Laboratory Press, Cold Spring Harbor, 1994.

17 Eilers, M., and Schatz, G., Binding of a specific ligand inhibits import of a purified precursor protein into mitocondria. Nature 322 (1986) 228-232.

18 Gambill, B.D., Voos, W., Kang, P.J., Miao, B., Langer, T., Craig, E.A., and Pfanner, N., A dual role for mitochondrial heat shock protein 70 in membrane translocation of preproteins. J. Cell Biol. 123 (1993) 119-126.

19 Gething, M.-J., and Sambrook, J., Protein folding in the cell. Nature 355 (1992) 33-45.

20 Glick, B.S., Wachter, C., Reid, G.A., and Schatz, G., Import of cytochrome $b_{7}$ to the mitochondrial imtermembrane space - the tightly folded heme-binding domain makes import dependent upon matrix ATP. Protein Sci. 2 (1993) 1901 1917.

21 Groot, G.S.P., Mason, T.L., and van Harten-Loosbroek, N., Varl is associated with the small ribosomal subunit of mitochondrial ribosomes in yeast. Molec. gen. Genet. I74 (1979) $339-342$ 
22 Hadikusumo, R.G., Meltzer, S., Choo, W.M., Jean-François, M.J., Linnane, A.W., and Marzuki, S., The definition of mitochondrial H + ATPase assembly defects in mit-mutants of Saccaromyces cerevisiae with a monoclonal antibody to the enzyme complex as an assembly probe. Biochim. biophys. Acta 933 (1988) 212-222.

23 Hartl, F.-U., Holdan, R., and Langer, T., Molecular chaperones in protein folding: the art of avoiding sticky situations. Trends biochem. Sci. 19 (1994) 20-25.

23a Hermann, J., Stuart, R.A., Craig, E.A., and Neupert, W:, Mitochondrial heat shock protein 70 , a molecular chaperone for proteins encoded by mitochondrial DNA. J. Cell Biol. 127 (1994), in press.

24 Höhfeld, J., and Hartl, F.-U., Requirement of the chaperonin cofactor Hspl0 for protein sorting in yeast mitochondria. J. Cell Biol. (1994), in press.

25 Hendrick, J.P., and Hart1, F.-U., Molecular chaperone functions of heat shock proteins. A. Rev. Biochem. 62 (1993) $349-384$

26 Hwang, S.T., and Schatz, G., Translocation of proteins across the mitochondrial inner membrane, but not into the outer membrane, requires nucleoside triphosphates in the matrix. Proc. natl Acad. Sci. USA 86 (1989) 8432-8436.

27 Ikeda, E., Yoshida, S., Mitsuzawa, H., Uno, I., and Toh-e, A., YGE1 is a yeast homologue of Escherichia coli grpE and is required for maintenance of mitochonfdrial functions. FEBS Lett. 39 (1994) 265-268.

28 Kang, P.-J., Ostermann, J., Shilling, J., Neupert, W., Craig, E.A., and Pfanner, N., Requirement for hsp70 in the mitochondrial matrix for translocation and folding of precursor proteins. Nature, Lond. 348 (1990) 137-143.

29 Kiebler, M., Becker, K., Pfanner, N., and Neupert, W., Mitochondrial protein import: specific recognition and membrane translocation of preproteins. J. Membrane Biol. 135 (1993) 191-207.

30 Kutejová, E., Durcová, G., Surofková, E., and Kuzela, S., Yeast mitochondrial ATP-dependent protease: purification and comparison with the homologous rat enzyme and the bacterial ATP-dependent protease La. FEBS Lett. 329 (1993) $47-50$.

31 Langer, T., Lu, C., Echols, H., Flanagan, J., Hayer, M.K., and Hartl, F.-U., Successive action of DnaK, DnaJ and GroEL along the pathway of chaperone-mediated protein folding. Nature, Lond. 356 (1992) 683-689.

32 Liberek, K., Marszalek, J., Ang, D., Georgopoulos, C., and Zylicz, M., Escherichia coli DnaJ and GrpE heat shock proteins jointly stimulate ATPase activity of DnaK. Proc. natl Acad. Sci. USA 88 (1991) 2874-2878.

33 Lindquist, S., and Craig, E.A., The heat shock proteins. A. Rev. Genet. 22 (1988) 631-677.

34 Manning-Krieg, U.C., Scherer, P.E., and Schatz, G., Sequential action of mitochondrial chaperones in protein import into the matrix. EMBO J. 10 (1991) 3273-3280.

35 Nelson R.J., Zeigelhoffer, T., Nicolet, C., Werner-Washburne, M., and Craig, E.A., The translation machinery and seventy kilodalton heat shock protein cooperate in protein synthesis. Cell 71 (1992) $97-105$.

36 Neupert, W., Hartl, F.-U., Craig, E.A., and Pfanner, N., How do polypeptides cross mitochondrial membranes? Cell 63 (1990) 447-450.

37 Ostermann, J., Horwich, A.L., Neupert, W., and Hartl, F.-U.,
Protein folding in mitochondria requires complex formation with hsp60 and ATP hydrolysis. Nature 341 (1989) 125-130.

38 Pelham, H.R.B., Speculations on the function of major heat shock and glucose-regulated proteins. Cell 46 (1986) 959-961.

39 Rassow, J., Hartl, F.-U., Guiard, B., Pfanner, N., and Neupert, W., Polypetides traverse the mitochondrial envelope in an extended state. FEBS Lett. 275 (1990) 190-194.

40 Rospert, S., Junne, T., Glick, B.S., and Schatz, G., Cloning and disruption of the gene encoding yeast mitochondrial chaperonin 10 , the homolog of $E$. coli groES. FEBS Lett. 335 (1993) 358-360.

41 Rowley, N., Prip-Buus, C., Westermann, B., Brown, C., Schwarz, E., Barrel, B., and Neupert, W., Mdj1p, a novel chaperone of the DnaJ family, is involved in mitochondrial biogenesis and protein folding. Cell 77 (1994) 249-259.

42 Schleyer, M., and Neupert, W., Transport of proteins into mitochondria: translocational intermediates spanning contact sites between outer and inner membranes. Cell 43 (1985) $339-350$.

43 Stuart, R.A., Gruhler, A., van der Klei, I.J., Guiard, B., Koll, H., and Neupert, W., The requirement of matrix ATP for the import of precursor proteins into the mitochondrial matrix and intermembrane space. Eur. J. Biochem. 220 (1994a) 9-18.

44 Stuart, R.A., Cyr, D.M., Craig, E.A., and Neupert, W., Mitochondrial molecular chaperones: their role in protein translocation. Trends biochem. Sci. 19 (1994) 87-92.

45 Suzuki, C.K., Suda, K., Wang, N., and Schatz, G., Requirement of the yeast gene LON in mitochondrial proteolysis and maintenance of respiration. Science 264 (1994) 273-276.

46 Terpstra, P., and Butow, R.A., The role of var1 in the asssembly of yeast mitochondrial ribosomes. J. Biochem. Biophys. 254 (1979) $12662-12669$.

47 Tzagoloff, A., and Myers, A.M., Genetics of mitochondrial biogenesis. A. Rev. Biochem. 55 (1986) 249-285.

48 Tzagoloff, A., and Dieckmann, C.L., PET genes of Saccharomyces cerevisiae. Microbiol. Rev. 54 (1990) 211-225.

49 van Dyck, L., Pearce, D.A., and Sherman, F., PIMI encodes a mitochondrial ATP-dependent protease that is required for mitochondrial function in the yeast Saccharomyces cerevisiae. J. biol. Chem. 269 (1994) 238-242.

50 Voos, W., Gambill, D.B., Guiard, B., Pfanner, N., and Craig, E.A., Presequence and mature portion of preproteins strongly influence the dependence of mitochondrial protein import on the heat shock 70 protein in the matrix. J. Cell Biol. 123(1993) $109-118$

51 Wang, N., Gottesman, S., Willingham, M.C., Gottesman, M.M., and Maruizi, M.R., A human mitochondrial ATP-dependent protease that is highly homologous to bacterial Lon protease. Proc. natl Acad. Sci. USA 90 (1993) 11247-11251.

52 Wachter, C., Schatz, G., and Glick, B.S., Protein import into mitochondria: the requirement for external ATP is precursorspecific whereas intramitochondrial ATP is universally needed for translocation into the matrix. Molec. cell. Biol. 5 (1994) in press.

53 Wagner, I., Arlt, H., van Dyck, L., Langer, T., and Neupert, W., Molecular chaperones co-operate with PIM1 protease in the degradation of misfolded proteins in mitochondria. EMBO J. 13 (1994), in press.

54 Xia, Z., and Matthews, F.S., Molecular structure of flavocytochrome $\mathrm{b}_{2}$ at $2.4 \mathrm{~A}$ resolution. J. molec. Biol. 212 (1990) $837-863$. 